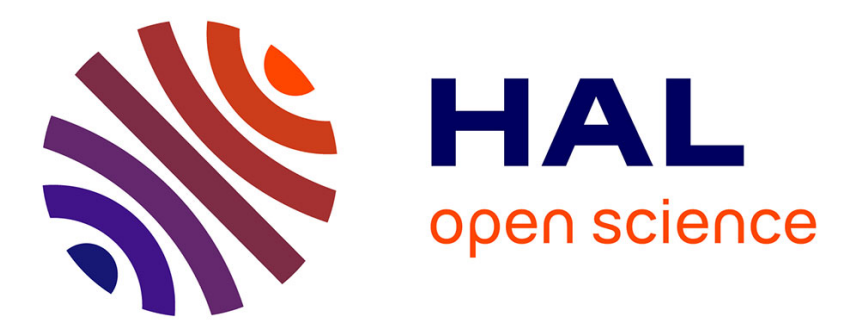

\title{
Modeling the spatial variability of the shear strength of discontinuities of rock masses: application to a dam rock mass
}

\author{
D. Sow, C. Carvajal, P. Breul, L. Peyras, P. Rivard, C. Bacconnet, G Ballivy
}

\section{- To cite this version:}

D. Sow, C. Carvajal, P. Breul, L. Peyras, P. Rivard, et al.. Modeling the spatial variability of the shear strength of discontinuities of rock masses: application to a dam rock mass. Engineering Geology, 2017, 220, pp.133-143. 10.1016/j.enggeo.2017.01.023 . hal-01581188

\author{
HAL Id: hal-01581188 \\ https://hal.science/hal-01581188
}

Submitted on 4 Sep 2017

HAL is a multi-disciplinary open access archive for the deposit and dissemination of scientific research documents, whether they are published or not. The documents may come from teaching and research institutions in France or abroad, or from public or private research centers.
L'archive ouverte pluridisciplinaire HAL, est destinée au dépôt et à la diffusion de documents scientifiques de niveau recherche, publiés ou non, émanant des établissements d'enseignement et de recherche français ou étrangers, des laboratoires publics ou privés. 


\title{
MODELING THE SPATIAL VARIABILITY OF THE SHEAR STRENGTH OF DISCONTINUITIES OF ROCK MASSES: APPLICATION TO A DAM ROCK MASS
}

4

\author{
Djibril SOW ${ }^{(1),(2),(3)}$; Claudio CARVAJAL ${ }^{(2)}$; Pierre BREUL ${ }^{(3)}$; Laurent PEYRAS $^{(2)}$; Patrice \\ RIVARD $^{(1)}$; Claude BACCONNET ${ }^{(3)}$; Gérard BALLIVY ${ }^{(1)}$ \\ ${ }^{(1)}$ Université de Sherbrooke, Sherbrooke, Québec, Canada, \\ \{ Djibril.Sow2; Patrice.Rivard; Gerard.Ballivy \} @USherbrooke.ca \\ ${ }^{(2)}$ Irstea, UR RECOVER, Aix-en-Provence, France \\ \{ laurent.peyras; claudio.carvajal \} @irstea.fr \\ (3) Université Blaise Pascal, Clermont Ferrand, France \\ \{Pierre.Breul; Claude.Bacconnet \}@polytech.univ-bpclermont.fr
}

\begin{abstract}
The shear strength of discontinuities plays a key role in the stability of rock masses, particularly in the case of analyzing the sliding stability of the rock foundations of gravity dams. This paper proposes a methodology for analyzing the spatial variability of shear strength along the joints of rock mass, based on the input parameters of the Barton and Choubey's model. The aim of this approach is to evaluate the reduction of the variance of the parameters involved at full-scale by identifying a deterministic trend varying in depth and a spatial correlation calculated from a variographic analysis. An advantage of this methodology is to use a simple experimental protocol (a laser profilometer, a portable shear test apparatus and a concrete sclerometer), which generates large sets of shear strength properties for assessing their spatial variability. The methodology is illustrated in the case of the rock foundation of a concrete gravity dam. Analysis of the spatial variability conducted for this case study led to a significant reduction of the variance of the variable analyzed. The advantage of this method is demonstrated through the evaluation of the probability of failure performed in a study of this structure's stability. Taking variance reduction into account in the case study led to significantly reducing the probability of failure assessed through a reliability analysis.
\end{abstract}

Keywords: rock joints, gravity-dam, rock foundation, shear strength, spatial variability, geostatistic. 


\section{INTRODUCTION}

The shear strength of discontinuities plays a key role in the stability of rock masses, particularly in the case of analyzing the sliding stability of the rock foundations of gravity dams. Rock masses often present vertical and horizontal variability regarding the shear strength properties of their joints (Phoon and Kulhawy 1999). The question of characterizing the spatial variability of the shear strength of rock masses appears crucial for providing a more satisfactory evaluation of their stability, since doing so would lead to reducing the variance of strength properties in limit state calculations and thus to optimizing the stability assessment. Indeed, taking into account the reduction in the variance of strength properties permits optimizing the estimation of: i) the values of conservative calculations for deterministic justifications, ii) the characteristic values corresponding to a fractile in semiprobabilistic justifications (Peyras et al., 2010), iii) the variance of random strength variables in probabilistic justification (Vanmarcke, 1983; Carvajal et al. 2009).

The variance of shear strength properties involved at the joint scale is lower than that evaluated at the laboratory scale. This reduction of variance results from the larger surface involved (linked to an average of punctual values) and from the spatial correlation of shear strength properties. Analyzing spatial variability through random fields makes it possible to take this reduced variance into account (Gravanis et al., 2014, Vanmarcke, 1983).

Several authors have focused on modeling the spatial variability of rock masses, such as (Duzgun et al., 2002), (Gravanis et al., 2014) and (Shamekhi et al., 2015). (Duzgun et al., 2002) proposed a probabilistic model to estimate the in-situ shear strength of rock joints based on shear strength measurements obtained in the laboratory. (Gravanis et al., 2014) put forward an analytical solution for the probabilistic evaluation of rock mass slope stability. The methodology proposed by (Gravanis et al., 2014) was based on random fields defined for the shear strength parameters of the Mohr-Coulomb criterion and was applied to the case of a joint along a predefined flat surface. (Shamekhi et al., 2015) also proposed a probabilistic method for evaluating the slope stability of a rock mass, in which the variability of the geometric properties of the joints were taken into account in a finite element model.

Modeling the spatial variability of a soil/rock strength property can be a difficult task because the number of test data obtained during site investigation is usually too sparse to carryout meaningful statistical analysis. Bayesian methods have been recently developed to address this issue in geotechnical and rock engineering (e.g., Wang and Cao 2013, Wang and Aladejare 2015, Wang et al. 2016).

Performing direct shear tests provides reference data for quantifying the shear strength of joints. However, these tests are expensive and only a limited number are performed with the test pieces available, which does not provide a statistically representative range of samples of the dispersion of shear strength properties. To solve this, we proposed a modified procedure using the Barton and Choubey's criterion (1977) to characterize joint shear strength (Sow et $a l ., 2015$ ). Our methodology is based on an experimental approach that uses simple resources to determine the parameters of the Barton and Choubey criterion: a laser profilometer, a portable shear test device and a concrete sclerometer. This experimental approach generates large sets of shear strength properties, through the application of a simple and inexpensive experimental protocol. The statistical sample of shear strength properties thus generated can then be analyzed and the spatial variability modeled.

The present paper continues in this direction in view to developing a methodology for analyzing the spatial variability of the shear strength of the joints of rock masses. The proposed methodology, based on the input parameters of the Barton and Choubey's model 
aims to evaluate the effects of reduced variance of the shear parameters involved in limit state calculations (Sow, 2015). An advantage of this methodology is to use a simple experimental protocol (a laser profilometer, a portable shear test apparatus and a concrete sclerometer), which generates large sets of shear strength properties for assessing their spatial variability.

The methodology proposed to analyze spatial variability comprises the following steps:

- The probabilistic description of the joints of a rock mass on the basis of a stereographic analysis;

- Using the Barton and Choubey criterion to characterize joint shear strength;

- Modeling spatial variability at the scale of the sample (from core samples) of parameters measured in the experimental campaign and the parameters of the Barton and Choubey criterion. Modeling the variability of the parameters studied highlights a deterministic trend as a function of spatial localization, a random measurement error variable and random error variable of the model linked to a deterministic trend;

- Modeling the spatial variability at the scale of the structure of the rock joint shear strength parameters on the basis of a variance reduction analysis.

The first two steps have already been used or published in the scientific literature to analyze rock masses and joint shear strength and are developed briefly in the first part of this paper. The second part of the paper presents the core of the methodological developments proposed to model the spatial variability of the joint shearing parameters. The methodology is illustrated in the third part of this article using the example of a rock foundation of a concrete gravity dam situated in Canada. Its efficiency is demonstrated through the presentation of a reliability analysis of the shear strength of a dam rock mass with a geo-modeling application using the finite elements method. The article ends with a discussion on the methodology and the results.

\section{MATERIAL AND METHODS}

This section briefly describes the tools and the methods already available in the literature that will be used in the methodology proposed in this paper in order to model the spatial variability of the shear strength of the discontinuities.

\subsection{Probabilistic description of the joints of a rock mass}

Rock masses are usually jointed heterogeneous media encompassing two types of element: rock blocks and joints. Due to their low stiffness, low strength regarding certain stresses (shear) and their hydraulic conductivity, joints play a predominant role in the behavior of rock masses (Panet, 1976).

The parameters that allow describing joint geometries are orientation, spacing and persistence (Blès, 1981).

The joints of a rock mass do not have a random orientation and are often organized in directional sets whose number is linked to the geological and mechanical phenomena involved during its formation and tectonic history (AFTES, 2003).

A stereographic projection was used for studying the distribution of joints in directional sets. When the different sets have been identified, we then analyze each set statistically by creating histograms of the distribution of geometric parameters relating to orientation, spacing and persistence (Hoek and Brown 1980, AFTES 2003). 
This probabilistic description of the joints of a rock mass provides the input data for the geomodelers and permits simulating the spatial distribution of the joints.

\subsection{Available data and the joint shear strength criterion}

The shear strength developed along the joints can be evaluated using two different approaches. The first entails direct shear testing while the second the use of an empirical failure criterion corresponding to a shear strength envelope (ISRM, 1978).

The shear strength evaluated by direct shear tests on rock joints is a reference experimental approach (Panet 1976, ASTM D5607-08 2008). However, the experimental procedures associated with these tests lead to obtain a limited number of samples available and therefore characterizing the shear strength variability of the rock studied.

The second approach uses empirical failure criteria and employs experimental protocols that are often quick and simple to use. The use of empirical failure criteria makes it possible to acquire shear data and get round the problem linked to the representativeness of the sampling (Grasselli and Egger, 2003). These empirical failure criteria include the Barton and Choubey's model (1977), which is recommended by the ISRM (1978) and remains widely used in engineering (Tatone and Grasselli 2012).

In our approach, the Barton and Choubey's criterion was used to characterize the joint shear strength. This criterion (equation [2]) introduces a parameter that takes roughness into account called JRC (Joint Roughness Coefficient), a mechanical strength parameter, JCS, that takes rock alteration into account (Joint Compressive Strength) and the residual friction angle, denoted $\varphi_{r}$. The term $\sigma_{n}$ represents the normal stress and $\tau_{p i c}$ the peak shear strength.

$$
\tau_{\text {pic }}=\sigma_{n} \tan \left(\varphi_{r}+J R C \log _{10}\left(\frac{J C S}{\sigma_{n}}\right)\right)
$$

The experimental protocols for evaluating Barton and Choubey (1977) parameters can be performed easily on almost every joint or test piece obtained from coring. The methodology chosen for our research is based on an experimental approach (Sow et al., 2015) which uses fewer resources in comparison to classical shear tests to determine the parameters of the Barton and Choubey criterion: a concrete sclerometer to evaluate the JCS parameter, a portable shear test device to determine $\varphi_{r}$ and a laser profilometer to scan the surface to evaluate the $J R C$.

The parameters measured directly by this experimental methodology are: i) the rebound values of altered and non-altered joints, $r$ and $R$ obtained using the Schmidt hammer; ii) the basic friction angle $\varphi_{b}$ obtained by shear testing of sawn joints; iii) the statistical roughness parameter $Z_{2}$ (likened to the average slope of the scanned profile).

At the end of the experimental campaign, the Barton and Choubey (1977) input parameters are evaluated using the following empirical models:

$$
\varphi_{r}=\left(\varphi_{b}-20\right)+20 * \frac{r}{R}
$$

$$
J C S=9.97 * \mathrm{e}^{(0.02 * R * \rho)} ; \text { with } \rho \text { : rock matrix density }
$$

$$
J R C=32,2+32,47 \cdot \log \left(Z_{2}\right)
$$


These empirical models [2, 3, 4] were proposed by Barton \& Choubey (1977) for $\varphi_{r}$, Deere \& Miller (1966) for JCS and Tse \& Cruden (1979) for JRC.

This methodology was subjected to a comparison with the results of measures performed with 35 direct shear tests (Sow et al., 2015), on altered and non-altered joints, tested under normal stresses varying from 0.15 to $2 \mathrm{MPa}$ (stresses generally found in dam foundations). In this comparison, the approach suggested by (Sow et al., 2015) led to a good estimation of the shear strength, with a small deviation (about 7\%) in comparison with direct shearing tests. It has the advantage of using relatively simple experimental protocols, making it possible to obtain sufficiently abundant data to then carry out a statistical analysis of spatial variability.

\subsection{Modeling spatial variability using geostatistical methods}

The purpose of a geostatistical approach is to model a random variable varying continuously in a fixed spatial domain (Matheron, 1970). With this approach, the characterization and modelling of the spatial variations of the variable studied are performed with variograms.

We consider a random variable $Z\left(\right.$.), a spatial domain $D$, and a sample of measures $Z\left(s_{1}\right)$, $Z\left(s_{2}\right), \ldots, Z\left(s_{\mathrm{n}}\right)$, where $s_{1}, s_{2}, \ldots, s_{\mathrm{n}}$ represent the spatial position or the site where the measures are performed. The variogram is a tool that characterizes the spatial dependence of the variable $Z($.) in domain $D$. The theoretical variogram $\gamma(h)$ is defined as:

$$
\gamma(h)=0.5 \operatorname{Var}[Z(s)-Z(s+h)]=0.5 E\left[(Z(s)-Z(s+h))^{2}\right]
$$

where $\operatorname{Var}$ and $E$ represent the variance and the expected value respectively, $s$ corresponds to the coordinates and $h$ is the distance between the points.

The nugget effect is defined as the value taken by the variogram $\gamma(h)$ when $h$ draws close to the origin. When $h$ increases, the variogram may or may not reach a plateau. Reaching a plateau indicates that counting from a certain distance, spatial dependence no longer exists between the data. This distance is called range and the term sill denotes the variance at which the plateau appears. This sill corresponds to the variance of variable $Z\left(\right.$.), also denoted $\sigma_{Z}^{2}$. The relation between the covariance function $C(h)$ and the variogram $\gamma(h)$ when it reaches a sill is the following:

$$
C(h)=\sigma_{Z}^{2}-\gamma(h)
$$

The variogram can be built experimentally from a sample of spatially localized measures. Finally, the experimental variogram can be fitted to the theoretical model. 


\section{THEORY AND CALCULATION}

This section presents the methodology proposed to model the spatial variability of shear strength parameters of rock joints. The approach used here to analyze the spatial variability of joint shear strength includes the following parameter modeling steps:

1. Modeling the variability of the parameters measured at the scale of the joints intersected by vertical boring;

2. Modeling the variability of the input parameters of the Barton and Choubey model, determined using an empirical model, at the scale of the joints intersected by boring;

3. Modeling the variability of the input parameters of the Barton and Choubey model integrated at the scale of the limit state (scale of the structure).

\subsection{Modeling the variability of the measured parameters}

\subsubsection{Definition and discretization of regionalized variables $Z_{i}(x)$}

We denote $Z_{i}(x)$ the regionalized variables corresponding to the parameters measured at the scale of the joints intersected by boring, where $x$ corresponds to the coordinate or vertical position in the borehole. It includes:

- the rebound value of the rock matrix $R(x)$ and the altered joints $r(x)$ obtained using a Schmidt hammer;

- the basic friction angle $\varphi_{b}(x)$ obtained by the shear test on the sawn joints;

- the statistical roughness parameter $Z_{2}(x)$ evaluated on the surfaces of the joints scanned with a laser profilometry.

In our approach to parameter variability modeling, we propose discretizing and formulating the regionalized variables $Z_{i}(x)$ as the sum of independent variables:

- a deterministic trend as a function of spatial localization $\mathrm{t}_{\mathrm{Zi}}(\mathrm{x})$;

- a variable of fluctuation around the non-biased trend $\mathrm{w}_{\mathrm{Z}_{\mathrm{i}}}(\mathrm{x})$;

- a random non-biased measurement error variable $\mathrm{e}_{\mathrm{Z}_{\mathrm{i}}}$ (Vanmarcke 1983, Phoon et al., 1999).

$$
\mathrm{Z}_{\mathrm{i}}(\mathrm{x})=\mathrm{t}_{\mathrm{Z}_{\mathrm{i}}}(\mathrm{x})+\mathrm{w}_{\mathrm{Z}_{\mathrm{i}}}(\mathrm{x})+\mathrm{e}_{\mathrm{Z}_{\mathrm{i}}}
$$

\subsubsection{Modeling of the deterministic trend as a function of spatial localization $\mathrm{t}_{\mathrm{Zi}}(x)$}

We determine the trend $\mathrm{t}_{\mathrm{Zi}}(\mathrm{x})$ of a parameter (the rock matrix rebound value $R$ and altered joints $r$, the basic friction angle $\varphi_{b}$ and the statistical roughness parameter $Z_{2}$ ) with a linear regression on the quantitative data of $\mathrm{Z}_{\mathrm{i}}(\mathrm{x})$ according to the depth of the borehole and taking into account the qualitative data of observations of borehole logs. The aim is to consider that the variability of the properties of a rock mass is not totally random and that part of this variability can be explained by physical phenomena and represented by a deterministic trend. 
Author-produced version of the article published in Engineering Geology, 220, 2017, 133-143.

The original publication is available at http://www.sciencedirect.com/science

DOI: 10.1016/j.enggeo.2017.01.023

\subsubsection{Modeling the random measurement error variable $e_{\mathrm{Zi}}$}

We model the measurement errors $\mathrm{e}_{\mathrm{Z}_{\mathrm{i}}}$ as zero mean random variables and of non-zero variance (Phoon et al., 1999, Favre 2004). Quantifying the variance of the measurement error is closely linked to the experimental protocol associated with the parameter measurement. We characterize the measurement error intrinsic to the test by the variance of the results obtained from a representative number of runs of this experimental protocol on nominally identical samples.

The measurement of the rebound value $R$ and $r$ corresponds to the mean of 10 higher values $\left\{R_{10}\right\}$ or $\left\{r_{10}\right\}$ obtained from 20 Schmidt hammer impacts on the surface of a sample. The measurement error is associated with the uncertainty on the estimation of the mean of these 10 higher values. Thus we propose estimating the variance of the measurement error of $R$ and $r$ by the sampling error according to equation [8]:

$$
\operatorname{Var}\left[\mathrm{e}_{\mathrm{R}}\right]=\frac{\operatorname{Var}\left[\left\{R_{10}\right\}\right]}{10}
$$

To determine the basic friction angle $\varphi_{b}$, the technical recommendations state that shear tests should be carried out on a smooth joint with 8 steps of increasing normal stress (Gyenge et Herget 1977). We estimate the variance of the measurement error $\varphi_{b}$ by the variance of the angles associated with each of these steps.

The measurement error on parameter $Z_{2}$ obtained from the laser scans was omitted given the high precision provided by this device.

\subsubsection{Modeling the trend fluctuation variable $\mathrm{w}_{\mathrm{Zi}}(x)$}

We consider the random fluctuation $\mathrm{w}_{\mathrm{Z}_{\mathrm{i}}}(\mathrm{x})$ as a zero mean variable and of non-zero variance (Phoon et al., 1999, Favre 2004). Its characterization consists in evaluating its variance and spatial correlation. We determine the variance of $\mathrm{w}_{\mathrm{Z}_{\mathrm{i}}}(\mathrm{x})$ from the variance of fluctuation $\left[\mathrm{Z}_{\mathrm{i}}(\mathrm{x})-\mathrm{t}_{\mathrm{z}_{\mathrm{i}}}(\mathrm{x})\right]$ and the variance of the measurement error $\mathrm{e}_{\mathrm{Z}_{\mathrm{i}}}$ :

$$
\operatorname{Var}\left[\mathrm{w}_{\mathrm{Z}_{\mathrm{i}}}(\mathrm{x})\right]=\operatorname{Var}\left[\mathrm{Z}_{\mathrm{i}}(\mathrm{x})-\mathrm{t}_{\mathrm{Z}_{\mathrm{i}}}(\mathrm{x})\right]-\operatorname{Var}\left[\mathrm{e}_{\mathrm{Z}_{\mathrm{i}}}\right]
$$

We characterize the spatial correlation of $\mathrm{w}_{\mathrm{Z}_{\mathrm{i}}}(\mathrm{x})$ with the variance reduction coefficient $\Gamma_{\mathrm{Z}_{\mathrm{i}}}^{2}\left(\mathrm{~L}_{\mathrm{v}}\right)$. It is likely determined from the experimental variogram of the values of $\mathrm{w}_{\mathrm{Z}_{\mathrm{i}}}(\mathrm{x})$. However, it is impossible to determine on each measured value of $\mathrm{Z}_{\mathrm{i}}(\mathrm{x})$, the share proper to the test error $\mathrm{e}_{\mathrm{Z}_{\mathrm{i}}}$. It is nonetheless noteworthy that the measurement error is by definition, proper to the test and so it should not present a spatial correlation. Therefore we suggest calculating the variogram directly from the data $\left[\mathrm{w}_{\mathrm{Z}_{\mathrm{i}}}(\mathrm{x})+\mathrm{e}_{\mathrm{Z}_{\mathrm{i}}}\right]$.

\subsection{Modeling the variability of input parameters of the Barton and Choubey model at the scale of the joints intersected by a borehole}

We denote $\mathrm{Z}_{\mathrm{di}}(x)$ the input parameters of the Barton and Choubey model at the scale of the joints intersected by the borehole, corresponding to parameters $\{\operatorname{JRC}(x), \operatorname{JCS}(x)$ and $\left.\varphi_{r}(x)\right\}$. They are determined using an empirical model $\mathrm{T}_{\mathrm{i}}$ and measured parameters $\mathrm{Z}_{\mathrm{i}}(x)$. This empirical model $\mathrm{T}_{\mathrm{i}}$ introduces an error assumed to be free of bias $\varepsilon_{\mathrm{i}}$ on parameter $\mathrm{Z}_{\mathrm{di}}(x)$. However, this model error is difficult to characterize. It can be assessed by a regression analysis of the models proposed in the literature; however, detailed input data of 
these models is rarely available. The model error can also be assessed by comparing the values estimated by a model and the values obtained experimentally (which are rarely available in large numbers). Taking into account the model errors requires special developments, which constitute a goal to improving the methodology proposed in this article.

$$
\mathrm{Z}_{\mathrm{di}}(x)=\mathrm{T}_{\mathrm{i}}\left(\mathrm{Z}_{1}(x) ; \ldots ; \mathrm{Z}_{\mathrm{n}}(x) ; \varepsilon_{\mathrm{i}}\right)
$$

The empirical models used in our study to evaluate the input parameters of the Barton and Choubey criterion from the values measured in our experimental campaign are presented by equations [2] to [4].

Monte Carlo simulations or a Taylor series can be used to estimate the expected value and the variance of the input parameters of the Barton and Choubey model at the joint scale $\mathrm{Z}_{\mathrm{di}}(x)$. The latter is an approximation whose advantage is that it allows an analytical calculation of the expected value and the variance of variables $\mathrm{Z}_{\mathrm{di}}(x)$, which is more practical in engineering.

The following equations [11] to [13] present the analytical formulas for estimating the expected value $E[. .$.$] of the input parameters of the Barton and Choubey criterion at the scale$ of the joints obtained from Taylor series linearization:

$$
\begin{gathered}
\mathrm{E}[J R C(x)]=32.2+32.47 \log \left(\mathrm{t}_{Z_{2}}(x)\right) \\
\mathrm{E}[J C S(x)]=9.97 * e^{\left(0.02 * \mathrm{t}_{r}(x) * \rho\right)} \\
\mathrm{E}\left[\varphi_{r}(x)\right]=\left(\mathrm{t}_{\varphi_{b}}(x)-20\right)+20 * \frac{\mathrm{t}_{r}(x)}{\mathrm{t}_{R}(x)}
\end{gathered}
$$

The equations [14] to [19] present the analytical formulas for estimating the variances $\operatorname{Var}[\ldots]$ of the input parameters of the Barton and Choubey criterion at the scale of the joints obtained from Taylor series linearization:

$$
\begin{gathered}
\operatorname{Var}[J R C(x)]=\left(\frac{32.47}{\ln (10) * \mathrm{t}_{Z_{2}}(x)}\right)^{2} \operatorname{Var}\left[\mathrm{w}_{Z_{2}}(x)\right] \\
\operatorname{Var}[J C S(x)]=\left(0.2 * \rho * e^{\left[0.02 * \rho * \mathrm{t}_{r}(x)\right]}\right)^{2}\left(\operatorname{Var}\left[\mathrm{w}_{r}(x)\right]+\operatorname{Var}\left[\mathrm{e}_{r}\right]\right) \\
\operatorname{Var}\left[\varphi_{r}(x)\right]=\operatorname{Var}\left[\mathrm{w}_{\varphi_{r}}(x)\right]+\operatorname{Var}\left[\mathrm{e}_{\varphi_{r}}\right]
\end{gathered}
$$

with:

$$
\begin{gathered}
\operatorname{Var}\left[\mathrm{w}_{\varphi_{r}}(x)\right]=\operatorname{Var}\left[\mathrm{w}_{\varphi_{b}}(x)\right]+a_{1}(x)^{2} \cdot \operatorname{Var}\left[\mathrm{w}_{R}\right]+a_{2}(x)^{2} \cdot \operatorname{Var}\left[\mathrm{w}_{r}(x)\right]-a_{3}(x) \cdot \\
\operatorname{Cov}\left(\mathrm{w}_{R}(x) ; \mathrm{w}_{r}(x)\right) \\
\operatorname{Var}\left[\mathrm{e}_{\varphi_{r}}\right]=\operatorname{Var}\left[\mathrm{e}_{\varphi_{b}}\right]+\left[a_{1}(x)^{2}+a_{2}(x)^{2}-a_{3}(x)\right] \cdot \operatorname{Var}\left[\mathrm{e}_{R}\right]
\end{gathered}
$$

Where:

$$
a_{1}(x)=\frac{20 * \mathrm{t}_{r}(x)}{\mathrm{t}_{R}(x)^{2}} ; a_{2}(x)=\frac{20}{\mathrm{t}_{R}(x)} ; a_{3}(x)=\frac{800 * \mathrm{t}_{r}(x)}{\mathrm{t}_{R}(x)^{3}}
$$




\subsection{Modeling the variability of the input parameters of the Barton and Choubey model at the scale of the limit state}

The geotechnical parameter to consider in the analysis of a limit state is that which is integrated on the entire area mobilized by the failure mechanism (Favre 2004). We denote $Z_{L}(L)$ the input parameters of Barton and Choubey's model integrated at the scale of the limit state (scale of the structure) along the portion of the borehole (portion with length $L$ ) affected by the limit state: $\left\{J R C_{L}(L) ; J C S_{L}(L) ; \varphi_{r_{L}}(L)\right\}$, with:

$$
\mathrm{Z}_{\mathrm{L}}(\mathrm{L})=\frac{1}{\mathrm{~L}} \int_{0}^{\mathrm{L}} \mathrm{Z}_{\mathrm{di}}(\mathrm{x}) \cdot \mathrm{dx}
$$

The following equations [21] à [23] present the analytical formulas for estimating the expected value $E[\ldots]$ of the input parameters of the Barton and Choubey criterion at the scale of the structure, obtained by Taylor series linearization:

$$
\begin{gathered}
\mathrm{E}\left[J R C_{L}(L)\right]=32.2+32.47 \frac{1}{L} \int_{0}^{L} \log \left(\mathrm{t}_{Z_{2}}(x)\right) \cdot d x \\
\mathrm{E}\left[J C S_{L}(L)\right]=\frac{1}{L} \int_{0}^{L} 9.97 * e^{\left(0.02 * \rho * \mathrm{t}_{r}(x)\right)} \cdot d x \\
\mathrm{E}\left[\varphi_{r_{L}}(L)\right]=\frac{1}{L} \int_{0}^{L}\left[\left(\mathrm{t}_{\varphi_{b}}(x)-20\right)+20 * \frac{\mathrm{t}_{r}(x)}{\mathrm{t}_{R}(x)}\right] \cdot d x
\end{gathered}
$$

We propose a geostatistical analysis to determine the variance reduction coefficient from the variograms obtained with regionalized variables, so the variance of the different input parameters of the Barton and Choubey's model can be determined at the scale of the limit state.

Equation [24] allows estimating the variance of variable $J C S_{L}(L)$ :

$$
\operatorname{Var}\left[J C S_{L}(L)\right]=\left(\frac{1}{L} \int_{0}^{L} 0.2 * \rho * e^{\left[0.02 * \rho * \mathrm{t}_{r}(x)\right]} \cdot d x\right)^{2} *\left(\Gamma_{r}^{2}(L) * \operatorname{Var}\left[\mathrm{w}_{r}(x)\right]+\operatorname{Var}\left[\mathrm{e}_{r}\right]\right)
$$

- with $\Gamma_{r}^{2}(L)$ being the variance reduction coefficient of the fluctuation variable $\mathrm{w}_{r}(x)$ obtained from the variogram calculation.

The variance of variable $J R C_{L}(L)$ is estimated with equation [25] :

$$
\operatorname{Var}\left[J R C_{L}(L)\right]=\Gamma_{Z_{2}}^{2}(L) * \operatorname{Var}\left[\mathrm{w}_{Z_{2}}(x)\right] *\left[\frac{1}{L} \int_{0}^{L}\left(\frac{32.47}{\ln (10) * \mathrm{t}_{Z_{2}}(x)}\right) \cdot d x\right]^{2}
$$

- with $\Gamma_{Z_{2}}^{2}$ being the variance reduction coefficient of the fluctuation variable $\mathrm{w}_{Z_{2}}$ obtained from the variogram calculation.

Equation [26] permits estimating the variance of variable $\varphi_{r_{L}}(L)$ :

$$
\operatorname{Var}\left[\varphi_{r_{L}}(L)\right]=\operatorname{Var}\left[\mathrm{w}_{\varphi_{r L}}(L)\right]+\operatorname{Var}\left[\mathrm{e}_{\varphi_{r L}}(L)\right]
$$

- with: 


$$
\begin{aligned}
\operatorname{Var}\left[\mathrm{w}_{\varphi_{r L}}(L)\right] & =\Gamma_{\varphi_{b}}^{2}(L) * \operatorname{Var}\left[\mathrm{w}_{\varphi_{b}}(x)\right]+\Gamma_{R}^{2}(L) * \operatorname{Var}\left[\mathrm{w}_{R}(x)\right] \cdot\left[\frac{1}{L} \int_{0}^{L} a_{1}(x) \cdot d x\right]^{2}+\Gamma_{r}^{2}(L) \\
& * \operatorname{Var}\left[\mathrm{w}_{r}(x)\right] \cdot\left[\frac{1}{L} \int_{0}^{L} a_{2}(x) \cdot d x\right]^{2} \\
& -\operatorname{Cov}\left(\Gamma_{R}^{2}(L) \cdot \mathrm{w}_{R}(x) ; \Gamma_{r}^{2}(L) \cdot \mathrm{w}_{r}(x)\right) \frac{1}{L} \int_{0}^{L} a_{3}(x) \cdot d x
\end{aligned}
$$

$$
\begin{aligned}
\operatorname{Var}\left[\mathrm{e}_{\varphi_{r L}}\right]= & \operatorname{Var}\left[\mathrm{e}_{\varphi_{b}}\right]+\operatorname{Var}\left[\mathrm{e}_{R}\right] \cdot\left[\frac{1}{L} \int_{0}^{L} a_{1}(x) \cdot d x\right]^{2}+\Gamma_{r}^{2}+\operatorname{Var}\left[\mathrm{e}_{R}\right] \cdot\left[\frac{1}{L} \int_{0}^{L} a_{2}(x) \cdot d x\right]^{2} \\
& -\operatorname{Var}\left[\mathrm{e}_{R}\right] \frac{1}{L} \int_{0}^{L} a_{3}(x) \cdot d x
\end{aligned}
$$

It is noteworthy that the variograms allow obtaining variance reduction coefficients built for fluctuation values around the deterministic trend $\mathrm{Z}_{\mathrm{i}}(\mathrm{x})-\mathrm{t}_{\mathrm{z}_{\mathrm{i}}}(\mathrm{x})$. We consider this fluctuation variable to be free of bias and stationary, thereby permitting the use of variograms.

\section{CASE STUDY AND RESULTS}

\begin{tabular}{|c|c|c|c|c|c|}
\cline { 2 - 6 } \multicolumn{1}{c|}{} & \multicolumn{3}{c|}{ Orientation parameters } & \multicolumn{2}{c|}{ Spacing } \\
\hline Joint set & Mean dip $\left(^{\circ}\right)$ & $\begin{array}{c}\text { Mean } \\
\text { direction of } \\
\text { dip }\left(^{\circ}\right)\end{array}$ & $\begin{array}{c}\text { Parameter K } \\
\text { (Langevin- } \\
\text { Fisher law) }\end{array}$ & $\begin{array}{c}\text { Mean } \\
\text { spacing (m) }\end{array}$ & $\begin{array}{c}\text { Parameter } \lambda \\
\text { (exponential } \\
\text { law) }\end{array}$ \\
\hline Set 1 & 16 & 99 & 42 & 0.22 & 4.55 \\
\hline Set 2 & 85 & 172 & 40 & 0.84 & 1.20 \\
\hline Set 3 & 85 & 238 & 55 & 1.37 & 0.73 \\
\hline
\end{tabular}

Table 1 : Results of the statistical analysis of orientation and spacing parameters

\subsection{The rock mass studied and spatial data}

The case study analyzed is the rock mass of an existing gravity dam with a maximum height of $30 \mathrm{~m}$ above the natural terrain. The direction of the dam axis is $110^{\circ}$, i.e. close to an east-west strike.

The rock mass is biotite granite gneiss of subdomain M4 of the Mékinac group. Unit M4 is composed of gneiss and composite gneiss. The rock mass exhibits three sets of joints: a set of joints developed along the foliation (set 1) and two sub-vertical sets (set 2 and 3). The statistical analyses of these joints led to modeling the distribution of the orientation and spacing parameters using a Langevin-Fisher law and an exponential law, respectively. The results of this analysis are summarized in Table 1. 
Author-produced version of the article published in Engineering Geology, 220, 2017, 133-143.

The original publication is available at http://www.sciencedirect.com/science

DOI: 10.1016/j.enggeo.2017.01.023

Four vertical boreholes of about 8 meters deep in the rock mass were drilled. Samples of rocks and joints were recovered from these boreholes, referenced by depth. The following tests were conducted:

- 62 Schmidt hammer tests: 52 and 10 tests to determine the rebound value $R$ and $r$ respectively;

- 19 shear tests on smooth joints $\left(\varphi_{b}\right)$;

- 19 surface scans with a laser profilometer $\left(Z_{2}\right)$.

\subsection{Results relating to the spatial variability of shear strength parameters}

Here, we implement the proposed methodology to the case study in order to analyze the spatial variability of the shear strength properties of rock joints.

\subsubsection{Modeling the parameters measured}

Table 2 summarizes the statistical analysis of the parameters measured experimentally (without spatial variability into account):

\begin{tabular}{|l|c|c|c|c|}
\hline Parameter & $r$ & $R$ & $\phi_{b}$ & $Z 2$ \\
& {$[-]$} & {$[-]$} & {$\left[{ }^{\circ}\right]$} & {$[-]$} \\
\hline Mean & 42.97 & 50.29 & 32.47 & 0.184 \\
\hline Standard deviation & 5.39 & 3.89 & 1.98 & 0.036 \\
\hline Variation coefficient & 0.13 & 0.08 & 0.06 & 0.197 \\
\hline Variance & 29.03 & 15.14 & 3.93 & 0.001 \\
\hline
\end{tabular}

Table 2 : Statistical data of the measured parameters

17

18

19

The measurements of $r$ are on average lower than $R$ given the alteration, but they present greater dispersion.

Figure 1 shows the results of the parameters measured as a function of depth $x$ under the dam-rock interface and the representation of a trend $t_{\mathrm{Z}}(x)$ estimated by linear regression. 

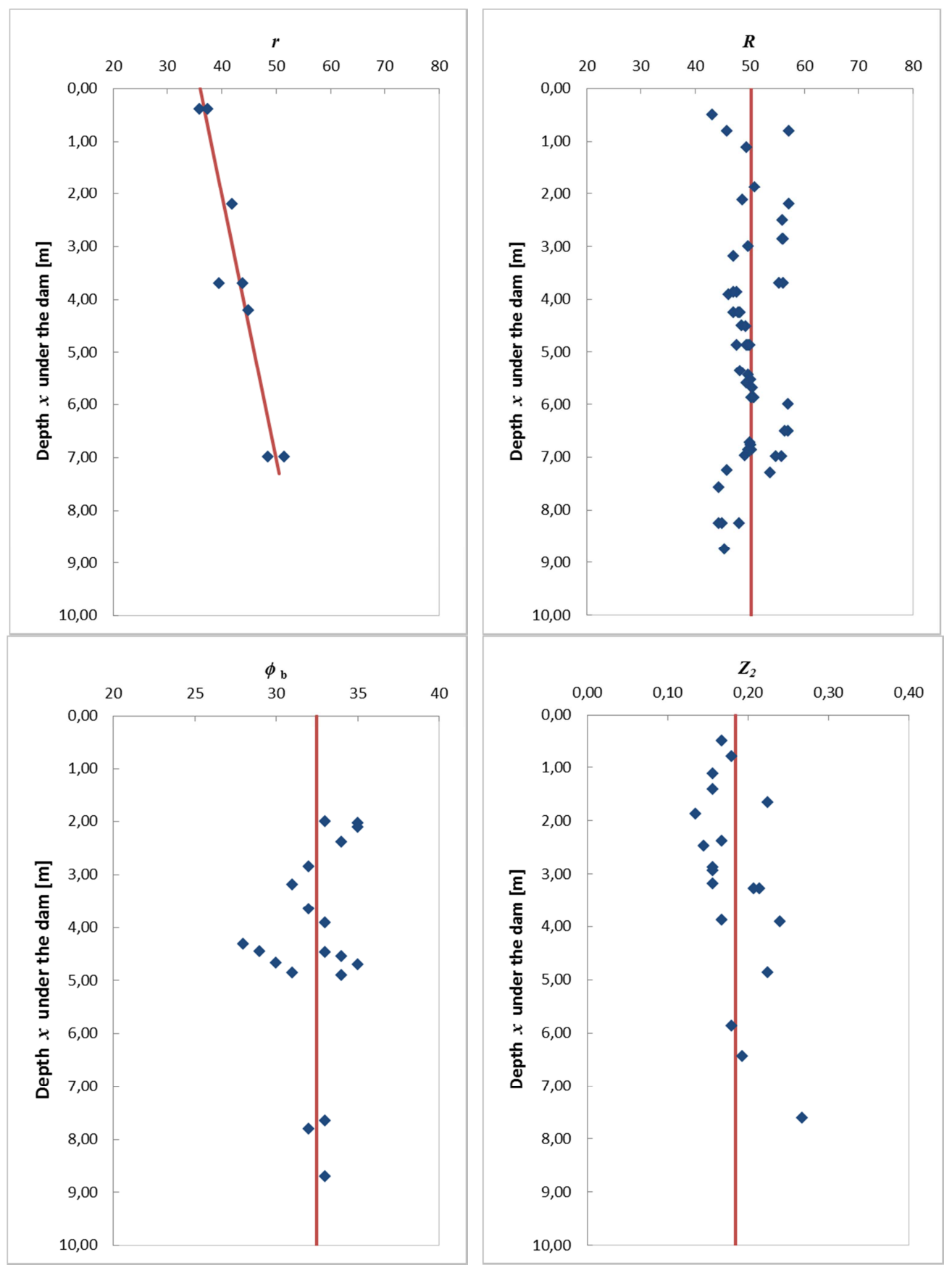

Figure 1: Parameters measured as a function of the depth under the dam-rock interface.

The data available for the rebound values of the altered joints $r(x)$ were less plentiful than the rebound values of the rock matrix $R(x)$. However, they allowed observing an increasing trend as a function of depth for the rebound values of the altered joints $r(x)$. This increase was observed down to a depth of about $6 \mathrm{~m}$ (limit of the data available on $r$ ) where the rebound value of the altered joints was close to the mean value observed for the joints of the rock matrix $R$.

For the other parameters (basic friction angle $\varphi_{b}$ and the statistical roughness value $Z_{2}$ ), we did not identify the net trend thus it was therefore reasonable to adopt a constant value equal to the mean for the deterministic trend $\mathrm{t}_{\mathrm{z}}(x)$. 
Author-produced version of the article published in Engineering Geology, 220, 2017, 133-143.

The original publication is available at http://www.sciencedirect.com/science

DOI: 10.1016/j.enggeo.2017.01.023

For each of the parameters measured Table 3 presents the variances of variables representing:

- the fluctuation or total deviation between the measured values and the deterministic trend $\left[\mathrm{Z}_{\mathrm{i}}(\mathrm{x})-\mathrm{t}_{\mathrm{Z}_{\mathrm{i}}}(\mathrm{x})\right]$;

- the measurement error $\mathrm{e}_{\mathrm{Z}_{\mathrm{i}}}$;

- the difference between the latter, $\mathrm{w}_{\mathrm{Zi}}(x)$.

\begin{tabular}{|c|c|c|c|c|}
\hline Parameter $\mathrm{Z}_{\mathrm{i}}$ & $\begin{array}{c}r \\
{[-]}\end{array}$ & $\begin{array}{c}R \\
{[-]}\end{array}$ & $\begin{array}{c}\phi_{b} \\
{\left[{ }^{\circ}\right]}\end{array}$ & $\begin{array}{c}\mathrm{Z} 2 \\
{[-]}\end{array}$ \\
\hline $\operatorname{Var}\left[\mathrm{Z}_{\mathrm{i}}(\mathrm{x})-\mathrm{t}_{\mathrm{Z}_{\mathrm{i}}}(\mathrm{x})\right]$ & 3.41 & 15.14 & 3.93 & 0.001 \\
\hline $\operatorname{Var}\left[\mathrm{e}_{\mathrm{Z}_{\mathrm{i}}}\right]$ & 0.59 & 0.59 & 0.005 & $\sim 0$ \\
\hline $\operatorname{Var}\left[\mathrm{w}_{\mathrm{Z}_{\mathrm{i}}}(\mathrm{x})\right]$ & 2.82 & 14.55 & 3.925 & 0.001 \\
\hline
\end{tabular}

Table 3 : Discretization of variances of measured parameters.

Variance of the fluctuation between the values measured and the deterministic trend $\operatorname{Var}\left[\mathrm{Z}_{\mathrm{i}}(\mathrm{x})-\mathrm{t}_{\mathrm{Z}_{\mathrm{i}}}(\mathrm{x})\right]$ :

By comparing the fluctuation variance values $\operatorname{Var}\left[\mathrm{Z}_{\mathrm{i}}(\mathrm{x})-\mathrm{t}_{\mathrm{Z}_{\mathrm{i}}}(\mathrm{x})\right]$ with those of table 2 (without taking spatial variability into account), we observe a reduction of variance only for parameter $r$, the only parameter for which a deterministic trend of evolution as a function of depth was identified.

Variance of the measurement error $\operatorname{Var}\left[\mathrm{e}_{\mathrm{Z}_{\mathrm{i}}}\right]$ :

For parameters $r$ and $R$, we evaluated the measurement error $\operatorname{Var}\left[\mathrm{e}_{\mathrm{Z}_{\mathrm{i}}}\right]$ for each of the 62 Schmidt hammer tests using equation [8] and we adopted the mean of these 62 values as representative of the measurement error $\operatorname{Var}\left[\mathrm{e}_{\mathrm{Z}_{\mathrm{i}}}\right]$ for $r$ and $R$.

For parameter $\varphi_{b}$, the low measurement error is due to the good alignment obtained during the shear tests on a smooth joint, and the experimental shear strength values as a function of normal stress.

We omitted the measurement error for $\mathrm{Z} 2$ given the precision of the profilometer, which appeared valid given the low variance of the fluctuation $\operatorname{Var}\left[\mathrm{Z}_{\mathrm{i}}(\mathrm{x})-\mathrm{t}_{\mathrm{Z}_{\mathrm{i}}}(\mathrm{x})\right]$.

Variance of the fluctuation $\operatorname{Var}\left[\mathrm{w}_{\mathrm{Z}_{\mathrm{i}}}(\mathrm{x})\right]$ :

Regarding the variance of the fluctuation $\operatorname{Var}\left[\mathrm{Z}_{\mathrm{i}}(\mathrm{x})-\mathrm{t}_{\mathrm{z}_{\mathrm{i}}}(\mathrm{x})\right]$, the share of the measurement error $\operatorname{Var}\left[\mathrm{e}_{\mathrm{Z}_{\mathrm{i}}}\right]$ remained low in comparison to the share of the residue corresponding to $\operatorname{Var}\left[\mathrm{w}_{\mathrm{Z}_{\mathrm{i}}}(\mathrm{x})\right]$.

\subsubsection{Modeling of the input parameters of the Barton and Choubey model at the scale of the joints intersected by a borehole}

The input parameters of the Barton and Choubey model at the scale of the joints intersected by a borehole were evaluated from measured parameters, using the empirical models of equations [2], [3] and [4]. 
Author-produced version of the article published in Engineering Geology, 220, 2017, 133-143.

The original publication is available at http://www.sciencedirect.com/science

DOI: 10.1016/j.enggeo.2017.01.023

Table 4 presents a synthesis of the statistical analysis of the input parameters of Barton and Choubey's model (without taking spatial variability into account):

\begin{tabular}{|l|c|c|c|}
\hline Parameter & $\begin{array}{c}J R C \\
{[-]}\end{array}$ & $\begin{array}{c}J C S \\
{[\mathrm{MPa}]}\end{array}$ & $\begin{array}{c}\varphi_{r} \\
{\left[{ }^{\circ}\right]}\end{array}$ \\
\hline Mean & 8.1 & 95 & 27.1 \\
\hline Standard deviation & 2.7 & 38 & 3.3 \\
\hline Variation coefficient & 0.33 & 0.40 & 0.12 \\
\hline Variance & 7.1 & 1444 & 10.6 \\
\hline
\end{tabular}

Table 4 : Statistical data of the input parameters of Barton and Choubey's model (without accounting for spatial variability).

Table 5 presents the results of applying the analytical formulas obtained by Taylor series linearization to estimate expected value (equations [11] to [13]) and variance (equations [14 to 19]) of the input parameters of Barton and Choubeys' criterion at the scale of the joints:

\begin{tabular}{|l|c|c|c|}
\hline Parameter, $Z_{\mathrm{di}}(x)$ & $J R C(x)$ & $J C S(x)$ & $\varphi_{r}(x)$ \\
\hline Expected value, $E\left[Z_{\mathrm{di}}(x)\right]$ & 8.1 & 129 & 29.2 \\
\hline Variance, $\operatorname{Var}\left[Z_{\mathrm{di}}(x)\right]$ & 7.1 & 326 & 4.2 \\
\hline
\end{tabular}

Table 5 : Expected value (mean) and Variance of the input parameters of Barton and Choubey's model at the scale of the joints.

The comparison of the variances of Table 4 with those of Table 5 permits highlighting the effects of reducing variance associated with a deterministic trend observed on the measured parameters.

This reduction of variance is zero for parameter $J R C(x)$, itself defined as a function of parameter $Z_{2}(x)$ which presents a trend that does not change as a function of depth in the borehole.

Parameters $\varphi_{r}(x)$ and $J C S(x)$ present reduced variance as they are defined as a function of the rebound value of the altered joints $r(x)$ which present an in increasing trend as a function of depth. This explains the evolution of the mean (or expected value) of parameters $\operatorname{JCS}(x)$ and $\varphi_{r}(x)$.

\subsubsection{Modeling the input parameters of the Barton and Choubey's model at the scale concerned by the limit state}

We consider a borehole length $L=10 \mathrm{~m}$ as representative of the scale concerned by the limit state (scale of the structure) for the input parameters of the Barton and Choubey's model.

The expected values $E[\ldots]$ of the input parameters of the Barton and Choubey's criterion at the scale of the structure are obtained directly from equations [21] to [23] defined as a function of the deterministic trend of the parameters measured. These values are presented in Table 7. 
Author-produced version of the article published in Engineering Geology, 220, 2017, 133-143.

The original publication is available at http://www.sciencedirect.com/science

DOI: 10.1016/j.enggeo.2017.01.023

To evaluate the variance $\operatorname{Var}[\ldots]$ of the input values of the Barton and Choubey model at the scale of the structure, it is necessary beforehand to build the variograms of the parameters measured to then obtain the variance reduction coefficient of the parameters at the scale of the structure (equations [24 to 28]).

Here, the case study is a gravity dam. For this type of dam, there is rarely more than one borehole along the same analyzed profile. Moreover, the distance between boreholes is generally of the same order of magnitude as the size of the area of the limit state considered (or even larger). All these conditions limit the interest of a variogram analysis in a horizontal direction. Thus, variograms were constructed along the vertical direction (corrected for inclined boreholes) which is also more practical as regards to the data available along a 11 borehole.

Figure 2 and Table 6 show the variograms obtained for the measured parameters:
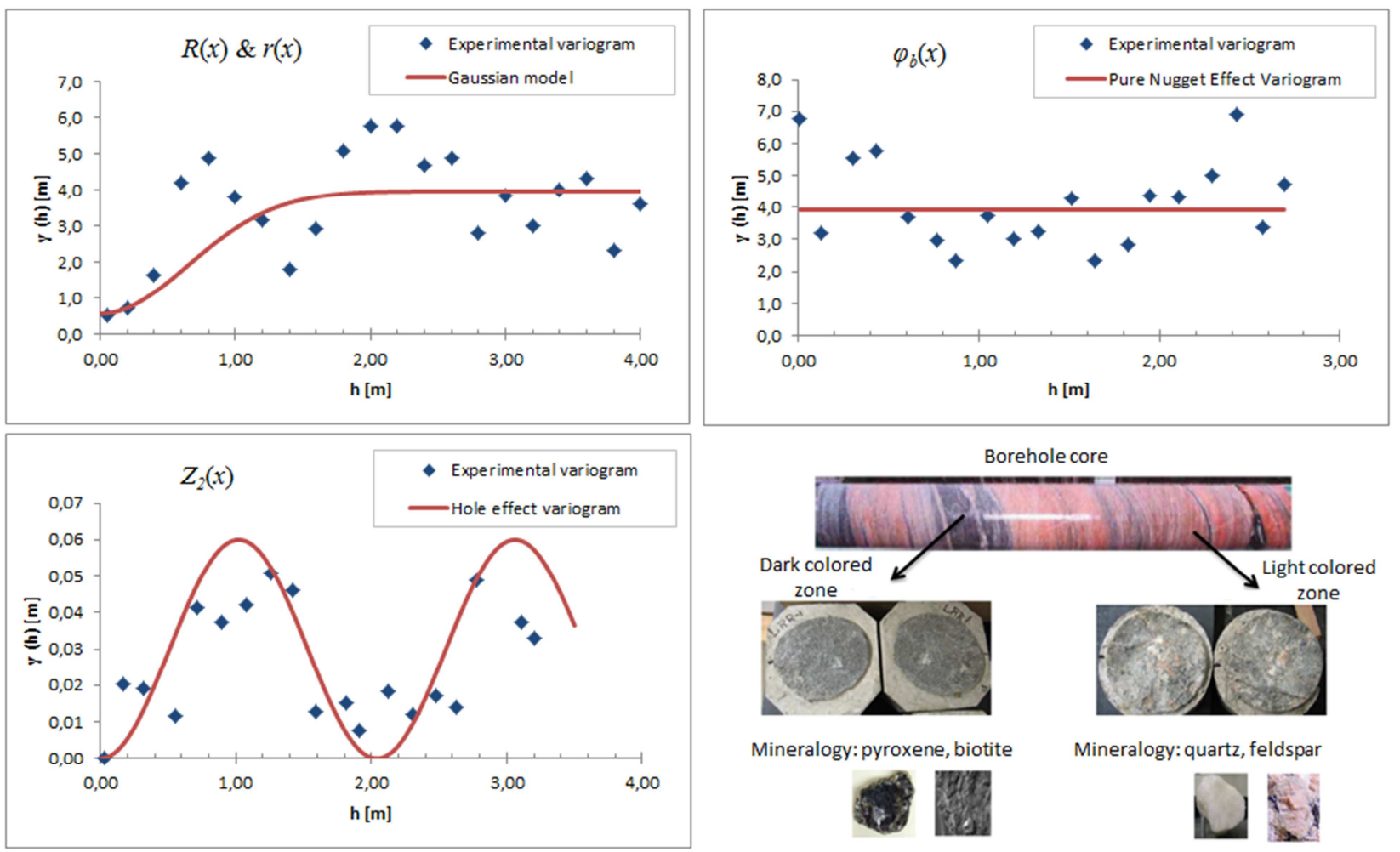

Figure 2: Variograms obtained for the parameters measured. 
Author-produced version of the article published in Engineering Geology, 220, 2017, 133-143.

The original publication is available at http://www.sciencedirect.com/science

DOI: 10.1016/j.enggeo.2017.01.023

\begin{tabular}{|l|l|c|l|}
\hline Parameter & \multicolumn{2}{|c|}{ Variogram model } & $\begin{array}{l}\text { Variograms } \\
\text { parameters }\end{array}$ \\
\hline$R(x) \& r(x)$ & Gaussian & $\gamma(h)=c \cdot\left(1-e^{-\left(\frac{h}{a}\right)^{2}}\right)+c_{0}$ & $\begin{array}{l}\mathrm{c}=3.4 \\
\mathrm{c}_{0}=0.59 \\
\mathrm{a}=0.92\end{array}$ \\
\hline$\varphi_{\mathrm{b}}(x)$ & Nugget & $\gamma(h)=c_{0}$ & $\mathrm{c}_{0}=3.93$ \\
$Z_{2}(x)$ & Hole effect & $\gamma(h)=c+\left(c_{0}-c\right) \cos \left(h \frac{\pi}{a}\right)$ & $\begin{array}{l}\mathrm{c}=0.03 \\
\mathrm{c}_{0}=0 \\
\mathrm{a}=1.02\end{array}$ \\
\hline
\end{tabular}

Table 6 : Variogram models of the measured parameters

Given the low number of $r(x)$ values, we aggregated them with the values of $R(x)$. This was justified knowing that it is the same type of measurement and that the variograms are built for the fluctuation between the measured values and the deterministic trend.

Part of the wave effect of the experimental variogram could be interpreted by the influence of the alternation of light-colored zones (richer in quartz-feldspar) and dark ones (richer in mafic minerals). This wave effect is increased for the roughness parameter $Z_{2}$, which may be due to the superficial nature of the laser profilometer measurements (the nature of the rebound measurements is less superficial given the propagation of impact energy in the sample).

It is noteworthy that when the mean of a variable varies as a function of depth, the field is no longer stationary. The variance reduction coefficient is therefore estimated for the fluctuation variable (which can be considered as stationary) between the values measured and the deterministic trend.

The variance reduction coefficients evaluated from the variograms are: $\Gamma_{r}^{2}(10 \mathrm{~m})=$ $\Gamma_{R}^{2}(10 \mathrm{~m})=0.15 ; \Gamma_{Z_{2}}^{2}(10 \mathrm{~m})=0.43 ; \Gamma_{\varphi_{b}}^{2}(10 \mathrm{~m})=1$.

Table 7 presents the results of applying the analytical formulas obtained by Taylor series linearization (and from the variograms) to estimate the expected value (equations [21 to 23]) and the variance (equations [23 to 27]) of the input parameters of the Barton and Choubey's criterion at the scale of the structure:

\begin{tabular}{|l|c|c|c|}
\hline Parameter, $Z_{\mathrm{Li}}(L)$ & $\begin{array}{c}J R C_{L}(L) \\
{[-]}\end{array}$ & $\begin{array}{c}J C S_{L}(L) \\
{[\mathrm{MPa}]}\end{array}$ & $\begin{array}{c}\varphi_{r L}(L) \\
{\left[{ }^{\circ}\right]}\end{array}$ \\
\hline Expected value, $E\left[Z_{\mathrm{Li}}(L)\right]$ & 8.1 & 129 & 29.2 \\
\hline Variance, $\operatorname{Var}\left[Z_{\mathrm{di}}(L)\right]$ & 2.9 & 107 & 4.2 \\
\hline
\end{tabular}

Table 7 : Expected value (mean) and Variance of the input parameters of the Barton and Choubey's model at the scale of the structure. 
Figure 3 summarizes the results obtained for the variance reduction analysis expressed as the standard deviation in order to keep the same unit to compare the parameters:
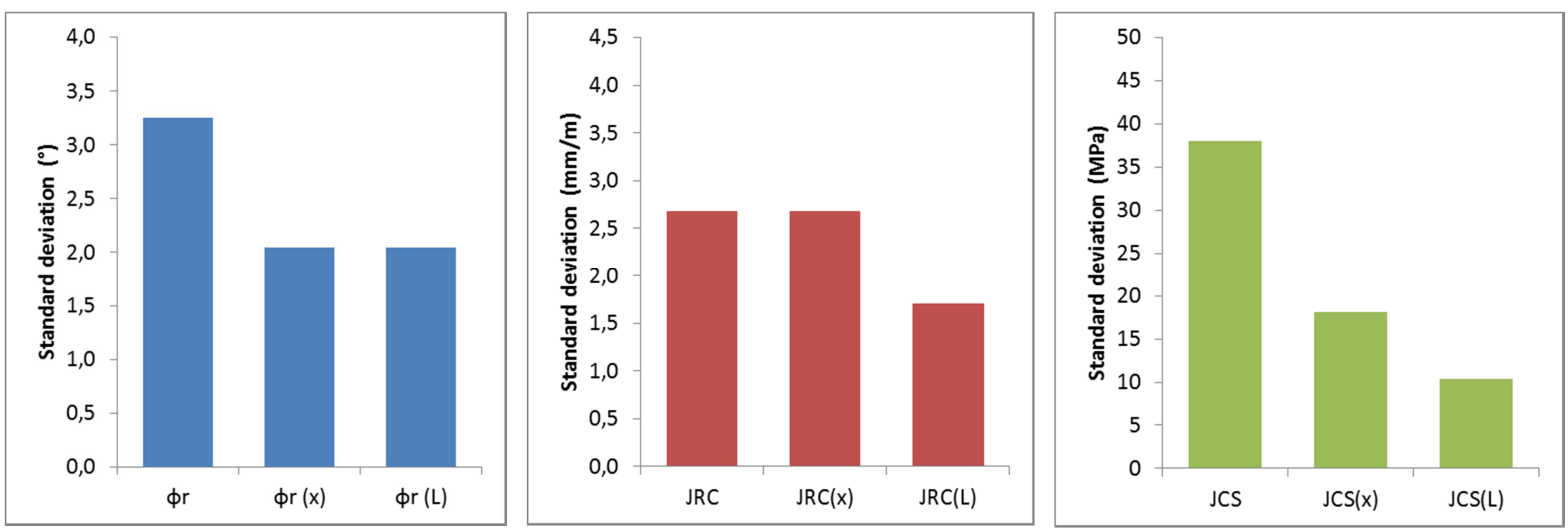

Figure 3: Reduction of the standard deviation of the Barton and Choubey parameters.

- the first term of each graph corresponds to the dispersion of the values without taking spatial variability into account;

- the second term corresponds to the dispersion of the fluctuation of values around the deterministic trend as a function of depth $(x)$;

- the third term is obtained by assigning the second term by the variance reduction coefficient evaluated for depth $(L)$.

\subsection{Results of application to the stability analysis}

In this application, Phase ${ }^{2} \odot$ geomodeler was used to build the geological model in 2D. Using a 2D model can be satisfactory in the case where the stability of gravity dam foundations is analyzed in 2D.

We focus on the limit state of shear strength on the joints of the rock mass. The condition of joint shear strength consists in verifying that the shear strength remains higher than the tangential stresses along the joints.

The Phase ${ }^{2} \odot$ geomodeler is based on the finite elements method and uses the Shear Strength Reduction (SSR) method to analyze this limit state of joint shear strength, and more generally, for analyzing the stability of rock masses (Griffits and Lane 1999).

For the reliability analysis, we use the probabilistic module integrated in the Phase $^{2}(\mathrm{C}$ geomodeler. It is defined in random variables and estimates the probability of failure using the Point-Estimate Method (Rosenblueth, 1975). This permits showing the impact that taking 
Author-produced version of the article published in Engineering Geology, 220, 2017, 133-143.

The original publication is available at http://www.sciencedirect.com/science

DOI: 10.1016/j.enggeo.2017.01.023

The probabilistic module of the Phase ${ }^{2} \odot$ software is limited to the Mohr-Coulomb model. The Barton and Choubey's model was then linearized in the range of normal stresses developed in the rock mass in order to implement Mohr-Coulomb's linear model.

In the application, we considered the shear strength parameters as random variables. Two sets of random variables were considered according to two configurations: with and without taking variance reduction into account. In order to facilitate the comparison between these two configurations, we considered only static actions taken as deterministic, including weight, the hydrostatic pressure and uplift (the latter without taking a drainage system into account).

Table 8 presents the probability laws considered to represent the random variables WITHOUT taking variance reduction into account.

\begin{tabular}{|l|l|l|l|}
\hline $\begin{array}{l}\text { Random variables WITHOUT taking } \\
\text { variance reduction into account }\end{array}$ & $\begin{array}{c}J R C \\
{[-]}\end{array}$ & $\begin{array}{c}J C S \\
{[\mathrm{MPa}]}\end{array}$ & $\begin{array}{c}\varphi_{r} \\
{\left[{ }^{\circ}\right]}\end{array}$ \\
\hline Type of probability law & Lognormal & Weibull & Weibull \\
\hline Mean & 8.1 & 104 & 28 \\
\hline Standard deviation & 2.7 & 29 & 2.9 \\
\hline
\end{tabular}

Table 8 : Random variables WITHOUT taking variance reduction into account.

To determine the type of probability law of the random variables used in the stability analysis, we used Monte-Carlo simulations: a probability distribution was fitted to the statistical data of the parameters measured $\left(R, r, \varphi_{b}\right.$ and $\left.Z_{2}\right)$ to then generate a sample comprising a high number of values for the input parameters of the Barton and Choubey model so as to finally fit a probability law to these parameters.

For the scale associated with the limit state, we considered a depth $(L)$ of $3.5 \mathrm{~m}$ given the width of the dam at its base and the dip of the joint set developed along the foliations. Table 9 presents the laws of probability considered to represent the random variables WITH the variance reduction taken into account (at the scale of the structure).

\begin{tabular}{|l|c|c|c|}
\hline $\begin{array}{l}\text { Random variables WITH the } \\
\text { variance reduction taken into } \\
\text { account }\end{array}$ & $\begin{array}{c}J R C_{L}(3,5 \mathrm{~m}) \\
{[-]}\end{array}$ & $\begin{array}{c}J C S_{L}(3,5 \mathrm{~m}) \\
{[\mathrm{MPa}]}\end{array}$ & $\begin{array}{c}\varphi_{r L}(3,5 \mathrm{~m}) \\
{\left[{ }^{\circ}\right]}\end{array}$ \\
\hline Type of probability law & Lognormal & Weibull & Weibull \\
\hline Mean & 8.1 & 85.7 & 26.9 \\
\hline Standard deviation & 1.8 & 6.5 & 1.99 \\
\hline
\end{tabular}

Table 9 : Random variables WITH the variance reduction taken into account

It is noteworthy that the values of Table 8 and Table 9 differ from those of Table 5 and Table 7, respectively. These differences essentially stem from the depth $(L)$ used to represent the scale associated with the limit state or scale of the structure $(L=10 \mathrm{~m}$ to illustrate the method proposed and $L=3.5 \mathrm{~m}$ for the specific application to the stability analysis);

Figure 4 shows the result of a simulation of joints in a rock mass and the displacements obtained. 


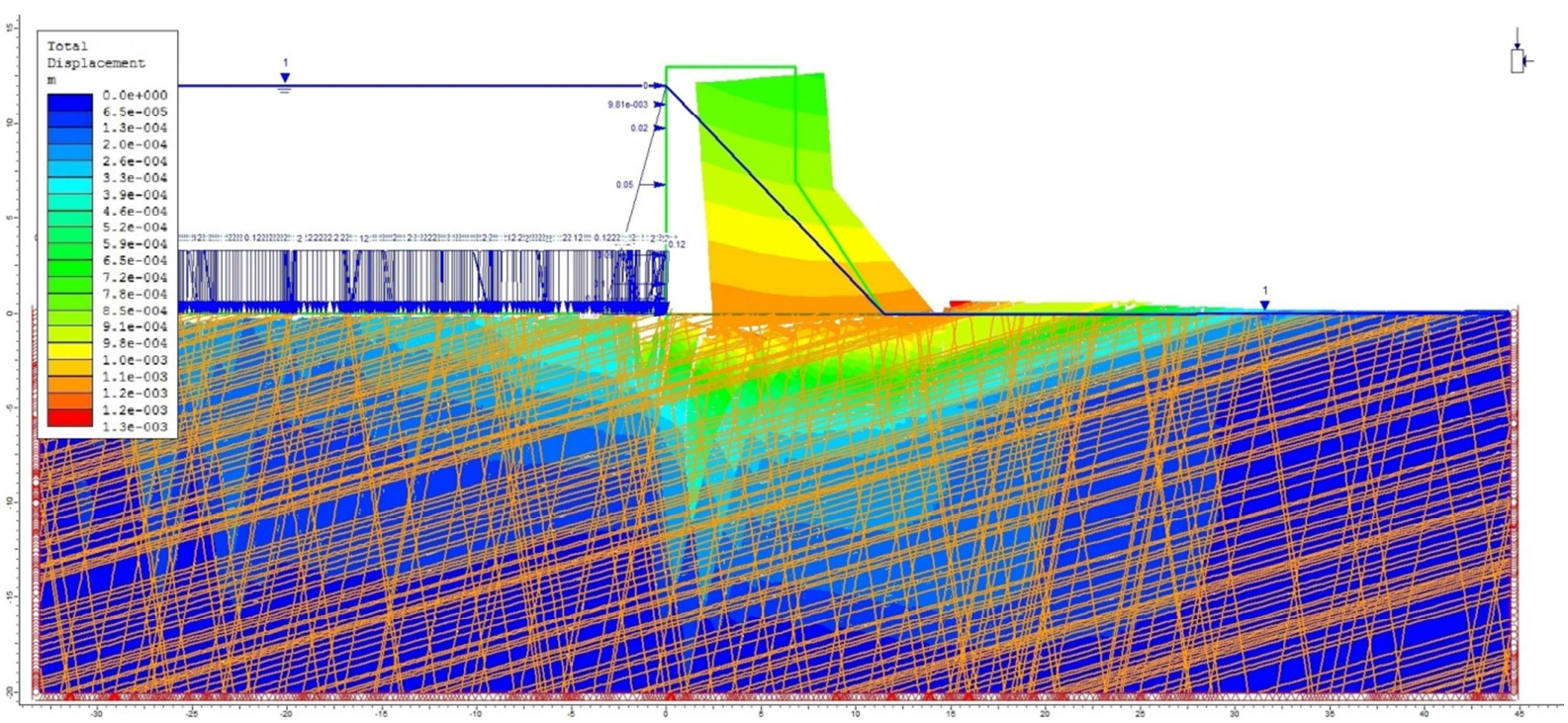

Figure 4 : Results of a simulation of rock mass joints and the associated displacement field.

The probability of failure evaluated for the case of random variables WITHOUT taking into account variance reduction is $2 \times 10^{-6}$. For this study, taking variance reduction into account led to reducing the probability of failure by several orders of magnitude $\left(<10^{-8}\right)$. Thus this application permits highlighting the impact that taking variance reduction (and the associated variance reduction) into account can have on the probability of failure evaluated in a reliability analysis.

\section{DISCUSSION ON THE METHODOLOGY AND ON THE RESULTS}

\subsection{Discussion on the methodology}

The methodology proposed permits analyzing the spatial variability of shear strength along the joints of rock masses. In particular it allows taking into account the effects of variance reduction by identifying a deterministic trend varying in depth and by analyzing spatial correlation on the basis of geostatistical analysis. Taking into account of the variance reduction of the shear parameters involved at the limit-state scale (scale of the structure) can lead to reduce the probability failure commonly assessed by a reliability analysis.

The methodology proposed is based on the Barton and Choubey's criterion that brings into play a greater amount of data than an approach based only on direct shear tests. Indeed, the methodology proposed is founded on an experimental approach leading to the generation of large sets of shear strength properties, using a simple and inexpensive experimental protocol (employing a laser profilometer, a portable shear test apparatus and a concrete sclerometer). The statistical sample of shear strength properties thus generated can then be subjected to an analysis and spatial variability modeling.

The proposed methodology uses an approximation by Taylor series linearization for estimating the expected value and the variance of the input parameters of the Barton and Choubey criterion. The accuracy of this approximation is conditioned by the linearity of the concerned formulas. However, this approximation has the advantage to allow an analytical 
calculation of the expected value and the variance of the variables analyzed, which is more practical in engineering.

The evaluation of shear strength requires the evaluation of a larger number of measured parameters and the use of empirical models to evaluate the parameters of the Barton and Choubey's criterion. Thus the study focused on identifying and characterizing the weight of the measured parameters on the evaluated parameters (parameters of the Barton and Choubey's criterion). For each parameter, the methodology suggested sought to separate the uncertainties that could be explained by spatial localization (with a deterministic trend) from the uncertainties linked to the measurements and from those linked to the models used.

The study also allowed taking into account an effect of scale by integrating measurement variability (done at the scale of the joints intersected by the borehole) in a larger volume associated with the limit state concerned (or the scale of structure). However, the effect of physical scale associated with the size of the samples was not considered in this methodology and therefore provides a future path for this work. In particular, it will require the utilization of experimental resources making it possible to carry out tests on much larger rock surfaces than those tested in the boreholes.

\subsection{Discussion on the results}

The case study analyzed demonstrated the applicability of the method proposed using real experimental data and for a rock mass foundation of a real dam.

The number of test data available in the case study is relatively large, but it might not be sufficient for an accurate statistical or geostatistical analysis. Despite the statistical uncertainty which may be generated by the number of experimental data available, the application of the proposed method can yield a reduction of the variance of the variable analyzed by taking into account the spatial variability.

Regarding the case study, it was possible to quantify the measurement error for the rebound tests. The laser profilometer provided a very high level of precision for the roughness measurements, which led to considering the raw measurement error as negligible. Nonetheless, the impact of the protocol used to obtain the roughness measurements could be analyzed by considering several possible profiles of the same sample.

The model error was not quantified in the case study. It is possible that the model error is more significant for the estimation of $\varphi_{r}$ and of JCS, with empirical models determined as a function of $\varphi_{b}, R$ and $r$ for $\varphi_{r}$ and as a function of $R$ only for $J C S$. The model error for $J R C$ is probably less significant $\left(Z_{2}\right.$ measured quantitatively on roughness profiles given by the Barton and Choubey, 1977). Nevertheless, the methodology proposed permitted incorporating these errors in every case when they were quantified.

Taking spatial evolution into account (in this case as a function of depth) via a deterministic trend led to a significant reduction of the variance of the variable analyzed. Regarding the case study, this trend was described only for the rebound value of the altered joints $r(\mathrm{x})$, leading to a significant reduction of the variance associated with variables $\varphi_{r}$ and $J C S$. Taking into account the spatial correlation allowed obtaining an additional reduction of variance (more significant in the case when the contribution of the deterministic trend was slight).

The stability analysis performed for the rock mass corresponded to an initial estimation and served to provide an illustrative value. Indeed, several simplifying hypotheses were 
employed, such as 2D modeling of the massif which led to omitting the kinematics of blocks in $3 \mathrm{D}$, and modeling uplifts without taking into account a drainage system.

Therefore the stability calculation results cannot be retained as absolute values, since only the relative values between the results with and without taking variability into account should be considered. In spite of the hypotheses adopted, this stability analysis was a direct application of the results of the methodology proposed and permitted highlighting the considerable impact that taking variance reduction into account can have on estimating the probability of failure.

\section{CONCLUSIONS}

We developed an approach that analyzes the spatial variability of the shear strength of joints using the input parameters of the Barton and Choubey's model. This approach was based on using the georeferenced data of these parameters characterizing the same set of joints along a borehole. It allowed identifying spatial organization and evaluating the variance reduction of the parameters involved at large scale by identifying a deterministic trend varying in depth and a spatial correlation deduced from a variographic analysis.

To evaluate the pertinence and applicability of this approach, we applied it in the case study of a rock mass making up the rock mass of a gravity dam. In this application, we focused on a set of joints intersected by a vertical, oriented borehole.

Taking variance reduction into account in the case study led to significantly reducing the probability of failure. Given the simplified nature of the reliability analysis performed in this study, we consider performing a fuller reliability analysis using a numerical 3D model, taking into account the random character of hydraulic stresses, the direct use of the Barton and Choubey's criterion and the explicit inclusion of random fields.

\section{ACKNOWLEDGEMENTS}

This study was made possible thanks to the financial support of IRSTEA (France), the University of Sherbrooke (Canada) and the Natural Science and Engineering Research Council of Canada (NSERC). 
Author-produced version of the article published in Engineering Geology, 220, 2017, 133-143.

The original publication is available at http://www.sciencedirect.com/science

DOI: 10.1016/j.enggeo.2017.01.023

APPENDIX A: TEST DATA

Test data used in this article is summarized in Table A.1.

\begin{tabular}{|c|c|c|}
\hline Borehole & $\begin{array}{c}\text { Depth } \\
{[\mathrm{m}]}\end{array}$ & $\begin{array}{c}R \\
{[-]}\end{array}$ \\
\hline FVG2 & 3,00 & 49,80 \\
\hline FVG2 & 4,50 & 48,60 \\
\hline FVG2 & 4,25 & 47,95 \\
\hline FVG2 & 4,25 & 46,97 \\
\hline FVG2 & 4,25 & 48,30 \\
\hline FVG2 & 7,58 & 44,34 \\
\hline FVID1 & 2,20 & 41,94 \\
\hline FVD1 & 3,70 & 39,48 \\
\hline FVD1 & 3,70 & 43,89 \\
\hline FVD1 & 7,25 & 45,77 \\
\hline FVG2 & 8,25 & 44,97 \\
\hline FVD1 & 6,99 & 51,60 \\
\hline FVD2 & 0,39 & 48,50 \\
\hline FVD2 & 0,39 & 35,99 \\
\hline FVD2 & 4,21 & 44,97 \\
\hline
\end{tabular}

\begin{tabular}{|c|c|c|}
\hline Borehole & $\begin{array}{c}\text { Depth } \\
{[\mathrm{m}]}\end{array}$ & $\begin{array}{c}\varphi_{\mathrm{b}} \\
{\left[{ }^{\circ}\right]}\end{array}$ \\
\hline FVG2 & 2,00 & 33 \\
\hline FVG2 & 2,03 & 35 \\
\hline FVG2 & 2,85 & 32 \\
\hline FVG2 & 3,65 & 32 \\
\hline FVG2 & 4,45 & 29 \\
\hline FVG2 & 4,55 & 34 \\
\hline FVG2 & 4,70 & 35 \\
\hline FVG2 & 4,90 & 34 \\
\hline FVG2 & 7,65 & 33 \\
\hline FVG2 & 7,81 & 32 \\
\hline FVG2 & 8,70 & 33 \\
\hline FVG3 & 2,10 & 35 \\
\hline FVG3 & 2,38 & 34 \\
\hline FVG3 & 3,18 & 31 \\
\hline FVG3 & 3,91 & 33 \\
\hline FVG3 & 4,32 & 28 \\
\hline FVG3 & 4,47 & 33 \\
\hline FVG3 & 4,67 & 30 \\
\hline FVG3 & 4,85 & 31 \\
\hline
\end{tabular}

\begin{tabular}{|c|c|c|}
\hline Borehole & $\begin{array}{c}\text { Depth } \\
{[\mathrm{m}]}\end{array}$ & $\begin{array}{c}Z_{2} \\
{[-]}\end{array}$ \\
\hline FVG3 & 0,50 & 0,168 \\
\hline FVG3 & 0,80 & 0,180 \\
\hline FVG3 & 1,12 & 0,156 \\
\hline FVG3 & 1,41 & 0,156 \\
\hline FVG3 & 1,65 & 0,225 \\
\hline FVG3 & 2,38 & 0,168 \\
\hline FVG3 & 2,48 & 0,145 \\
\hline FVG3 & 1,87 & 0,135 \\
\hline FVG3 & 2,93 & 0,156 \\
\hline FVG3 & 2,87 & 0,156 \\
\hline FVG3 & 3,18 & 0,156 \\
\hline FVG3 & 3,27 & 0,214 \\
\hline FVG3 & 3,27 & 0,207 \\
\hline FVG3 & 3,91 & 0,240 \\
\hline FVG3 & 3,87 & 0,168 \\
\hline FVG3 & 4,87 & 0,224 \\
\hline FVG3 & 6,43 & 0,193 \\
\hline FVG3 & 5,87 & 0,180 \\
\hline FVG3 & 7,61 & 0,268 \\
\hline
\end{tabular}

\begin{tabular}{|l|l|l|}
\hline FVG3 & 3,87 & 47,70 \\
\hline FVG3 & 3,87 & 47,00 \\
\hline FVG3 & 4,52 & 49,30 \\
\hline FVG3 & 4,87 & 47,70 \\
\hline FVG3 & 5,37 & 48,20 \\
\hline FVG3 & 5,44 & 49,80 \\
\hline FVG3 & 4,87 & 49,80 \\
\hline FVG3 & 5,54 & 50,20 \\
\hline FVG3 & 5,59 & 49,40 \\
\hline FVG3 & 4,87 & 49,40 \\
\hline FVG3 & 5,69 & 50,50 \\
\hline FVG3 & 4,87 & 50,00 \\
\hline FVG3 & 5,87 & 50,40 \\
\hline FVG3 & 6,72 & 50,10 \\
\hline FVG3 & 6,77 & 50,20 \\
\hline FVG3 & 5,87 & 50,80 \\
\hline FVG3 & 6,87 & 49,90 \\
\hline FVG3 & 6,87 & 50,40 \\
\hline FVG3 & 6,97 & 49,20 \\
\hline FVG3 & 6,87 & 49,80 \\
\hline
\end{tabular}




\begin{tabular}{|l|l|l|} 
FVD1 & 0,80 & 57,20 \\
\hline FVD1 & 2,20 & 57,20 \\
\hline FVD1 & 2,50 & 56,00 \\
\hline FVD1 & 2,86 & 56,00 \\
\hline FVD1 & 2,86 & 56,20 \\
\hline FVD1 & 3,70 & 56,10 \\
\hline FVD1 & 3,70 & 55,40 \\
\hline FVD1 & 6,00 & 57,10 \\
\hline FVD1 & 6,50 & 57,10 \\
\hline FVD1 & 6,50 & 56,50 \\
\hline FVD1 & 6,99 & 55,80 \\
\hline FVD1 & 6,99 & 54,75 \\
\hline FVD1 & 7,30 & 53,77 \\
\hline
\end{tabular}

\section{REFERENCES}

AFTES (2003). «Recommandations relatives à la caractérisation des massifs rocheux utile à l'étude et à la réalisation des ouvrages souterrains ». Tunnels et Ouvrages souterrains $\mathrm{N}^{\circ} 177$ : 30p.

ASTM D5607-08 (2008). Standard Test Method for Performing Laboratory Direct Shear Strength Tests of Rock Specimens Under Constant Normal Force. ASTM International, West Conshohocken, PA, 2008.

Matheron G. (1970) La théorie des variables régionalisées et ses applications Cahiers du Centre de Morphologie Mathématiques de Fontainebleau Fascicule n 5, 212 pages.

Barton N.R. \& Choubey V. (1977). « The shear strength of rock joints in theory and practice », Rock Mech Rock Eng., vol. 10, n 1-2, 1977, p. 1-54.

Blès, J.-L. et Feuga B. (1981). La fracturation des roches. BRGM, Manuel et méthodes $\mathrm{n}^{\circ} 1$, vol.1, 160p. 1981.

Carvajal C., Peyras L., Bacconnet C., Bécue J-P (2009). «Probability Modelling of Shear Strength Parameters of RCC Gravity Dams for Reliability Analysis of Structural Safety”. European Journal of Environmental and Civil Engineering, 2009 vol.13 No.1, pp.91-119.

CFBR (2012). Recommandations pour la justification de la stabilité des barrages-poids. Comité Français des Barrages et Réservoirs, 2012.

Deere, D. U. and R. P. Miller (1966). Engineering classification and index properties for intact rock. Report AFWL-TR-65-116. Air Force Weapons Laboratory (WLDC). Kirtland Air Force Base. New Mexico 87117.

Duzgun, H.S.B., Yucemenb, M.S., Karpuzc, C. (2002). "A probabilistic model for the assessment of uncertainties in the shear strength of rock discontinuities". International Journal of Rock Mechanics \& Mining Sciences, vol.39, 2002, p. 743754. 
Favre J.L. (2004). Sécurité des ouvrages / Risques : Modélisation de l'incertain, fiabilité, analyse des risques, Paris, Ellipses, 2004.

Gyenge, M. and G. Herget (1977). Determination of strength properties of rock discontinuties by direct Shear test. Laboratory tests for design parameters. Canada, Canmet Report. 77-26.

Grasselli, G. and P. Egger (2003). "Constitutive law for the shear strength of rock joints based on three-dimensional surface parameters". International Journal of Rock Mechanics and Mining Sciences 40(1): 25-40.

Gravanis, E., Pantelidis, L., Griffiths, D.V. (2014). “An analytical solution in probabilistic rock slope stability assessment based on random fields". International Journal of Rock Mechanics \& Mining Sciences, vol.71, 2014, p. 19-24.

Griffiths, D.V. and Lane, P.A. (1999). "Slope stability analysis by finite elements". Geotechnique, vol. 49, no. 3, pp.387-403.

Hoek \& Brown (1980). Underground excavation in rock. London, Instn Min. Metall.

ISRM (1978). "Suggested methods for the quantitative description of discontinuities in rock masses". Int. J. Mech. Min. Sci \& Geomech. Abstr Vol 15: pp 319-368.

Jaboyedoff, M., F. Philippossian, et al. (1996). "Distribution spatiale des discontinuités dans une falaise, approche statistique et probabilistique". Short CourseQUANTERRA: International independant center of climate change impact and natural risk analysis in mountainous area: $13 \mathrm{p}$.

Merrien-Soukatchoff, V. and T. Korini (2011). "Use of an integrated discrete fracture network code for stochastic stability analyses of fractured rock masses" Rock Mechanics and Rock Engineering, 2012, Volume 45, Issue 2, pp 159-181.

Panet, M. (1976). La Mécanique des roches appliquée aux ouvrages de génie civil. Paris : Presses de l'Ecole Nationale des Ponts et Chaussées, 1976, 235p.

Peyras L., Merckle S., Royet P., Bacconnet C., Ducroux A. (2010). Study on a semiprobabilistic method for embankment hydraulic works - Application to sliding mechanism. European Journal of Environmental and Civil Engineering, Vol. 14, n5. pp.669-691

Phoon, K. K. and F. H. Kulhawy. (1999). "Characterization of geotechnical variability". Canadian Geotechnical Journal, vol.36, n4, p.612-624, 1999.

Rosenblueth, E. (1975) Point estimates for probability moments, in Proceedings of the National Academy of Sciences, Vol. 72 (10), pp. 3812-3814.

Shamekhi, E., Tannant, D.D. (2015). "Probabilistic assessment of rock slope stability using response surfaces determined from finite element models of geometric realizations". Computers and Geotechnics, vol.69, 2015, p. 70-81.

Sow, D. (2015). Variabilité spatiale de la résistance au cisaillement des discontinuités des fondations rocheuses de barrages. Université Blaise Pascal - Clermont-Ferrand II, 2015. PhD thesis, French.

Sow D, Rivard P, Peyras L, Breul P, Moradian Z.A., Bacconnet C, Ballivy G. (2015). Comparison of Joint Shearing Resistance Obtained with the Barton and Choubey Criterion and with Direct Shear Tests. Technical Note, Rock Mechanics and Rock Engineering, pp 1-5. 
Tse R., Cruden D.M. (1979). "Estimating joint roughness coefficients". International Journal of Rock Mechanics and Mining Sciences \& Geomechanics Abstracts. Vol.16, n5 (1979), pp. 303-307.

Vanmarcke, E. H. (1983). Random fields: Analysis and Synthesis (Revised and Expanded New Edition 2010). London: MIT Press, 372p., 1983. ISBN: 0262220261.

Wang Y, Cao Z. (2013). "Probabilistic characterization of Young's modulud of soil using equivalent samples". Engineering Geology 159 (2013), pp. 106-118.

Wang Y., Aladejare A.E. (2015). "Selection of site-specific regression model for characterization of uniaxial compressive strength of rock". International Journal of Rock Mechanics \& Mining Sciences 75 (2015), pp. 73-81.

Wang Y, Cao Z., Li D. (2016). "Bayesian perspective on geotechnical variability and site characterization". Engineering Geology 203 (2016), pp. 117-125. 